\title{
The Influence of Strength and Speed on the Performance of Fosbury Flop: A Theoretical Study
}

\author{
Dai wen ${ }^{1}$, Xu xiaolei ${ }^{2}$ \\ ${ }^{1}$ The Phisical Education Institute of Shan dong University, 250014, China \\ ${ }^{2}$ The Phisical Education Institute of Shan dong Normal University, 250014, China \\ xuxiaolei163@163.com
}

\begin{abstract}
Jumping ability is the comprehensive reflection of body strength, running speed, reaction speed, physical coordination, flexibility as well as agility, of which body strength and running speed play a vital important role in the development of jumping ability. Jumping ability is the basic guarantee for getting ideal performance of Fosbury Flop. Jumping ability reaches its maximum when body strength and running speed tend to be the case of perfect combination. To analyze the influence of improving physical strength and increasing running speed on jumping ability, references can be provided for Fosbury Flop athletes to improve their performance.

Index Terms - strength, speed, Fosbury Flop
\end{abstract}

\section{Introduction}

With the development of competitive sports, in order to be higher, faster and stronger, athletes continue to carry training. There are many aspects involved in Athletes' training, such as strength, speed, endurance, flexibility, coordination and so on, of which strength and speed training take up most of the time. Thus, it is obvious that strength and speed are valued in the athletes' performance. The degree of strength and speed vary in different sports. For example, in boxing, the development of strength is more emphasized with the premise that athletes' speed is guaranteed, while in, such as, hundred-meter track events, the acceleration of speed is more valued. This paper is exampled with high jump in athletics events to discuss which of the two factors of strength and speed is more helpful in improving the performance.

\section{The relationship of strength and speed}

Speed indicates how fast the object moves, while strength means physical energy. Strength and speed are crucial in sporting events.

Firstly, as we all know, speed is proportional to strength as for the objects of equal quality. A material about football says: "The strength of kicking the ball depends on the amplitude of the swing angle of the thigh, while the speed of the ball depends on the swing speed of the shank." In physics there is a formula says: $\mathrm{Ft}=\mathrm{mV}$. An impression is made that if the destructive power of the ball is great, it will be thought that it is because the strength of kicking the ball that is great, which, thus, leads to the fast speed of the ball. Is it rational to conceive that the greater the force that is made on an object is, the faster its speed will be? To begin with, if the force of 100 newtons is made on a tractor which weighs 1 ton, the tractor will still be static. Second, to add 50 newtons' force on a shot put weighing 5 kilograms and the time exerting upon the shot put is 2 seconds, the speed of the shot put will be 20 meters per second when it is thrown. Then when the force of pushing the shot put is added to 100 newtons and the time exerting on it is 1 second, still, the speed of the shot put is 20 meters per second. Thus, it is obvious to see that there is no direct relation between speed and force. Instead, speed is related to momentum. As for the object of the same weigh, the faster the speed is, the greater the momentum will be, while as for the object of the same speed, the more the weigh is, the greater the momentum will be, and vice versa.

Secondly, although there's no direct relation between speed and strength, in biomechanics strength is the assurance to speed. There's no speed without strength. The speed of the physical movement derives from the strength gained when the muscles contract. Exercise physiology shows that when the concentrations of calcium in cytosol increases to $10 \mathrm{mols}$ per litre, calcium will integrate with $\mathrm{TnC}$. Then the conformational changes of TnC happen, which enhance the binding force of $\mathrm{TnC}$ with $\mathrm{TnI}$ and $\mathrm{TnT}$. The combination of $\mathrm{TnC}, \mathrm{TnI}$ and $\mathrm{TnT}$ impairs the binding force of TnI and actin, which causes the actin to break away from $\mathrm{TnI}$ and go to the started state. At the same time, TnT makes tropomyosin move deep into the spiral groove of the actin so that there is no possibility for actin to integrate with myosin. Then, actin combines with the head of the myosin, forming the new actin that is with cross-bridge. This new actin greatly improves the enzymatic activity of myosin's ATP, and myosin catalyses ATP which causes the hydrolysis reaction to take place. The energy that is released when the hydrolysis reaction happens forces the cross-bridge to change its angle, and the energy that is released by the hydrolysis reaction helps the cross-bridge to resume its position to combine with another ATP. So on ad infinitum, the muscles contract which causes the movement of the body and then speed is produced. Strength and speed has a causal relationship in the physiology.

Finally, strength and speed are two of the main factors that indicate physical fitness. To take part in all kinds of athletic events, athletes should have not only good strength quality, but also good speed quality, and none can be dispensed with. In order to achieve ideal performance, athletes should realize the perfect combination of strength and speed and the combination of strength and speed for Fosbury Flop athletes is more demanding. Strength and speed are inseparable in sports science. 


\section{The differences and the developmental trend of the take-off movements in modern Fosbury Flop}

High jump, as a competitive event, originated from Britain in the 18th century. In 1864, a British man named Cosi first thought up the leap-type high jump. The athletes adopted this type of high jump in the first modern Olympic Games, and won the championship by 1.8 meter. Foluo Zuff, an athlete from Soviet Union, created straddle high-jump in 1923, which is of great significance. In 1978, Jas shi CenKe, also from Soviet Union, gained a good score by 2.35 meter in this posture. However, in present national competition only the back style high-jump that is invented by Fosbury can be seen. A debate on whether the straddle high-jump or the back style high-jump was better lasted for a long time in sports world. It was until 1980s that the back style high-jump replaced the straddle high-jump. Different athletes adopt different type of take-off movements in the back style high-jump. Some athletes prefer one-armed take-off, while some others both. Take the finals of high-jump in the Olympic Games of Sydney and Beijing for examples:

TABLE 1. The comparison on take-off movements and scores

\begin{tabular}{lcc}
\hline & $\begin{array}{r}\text { Olympic Games } \\
\text { in Sydney, 2000 }\end{array}$ & $\begin{array}{c}\text { Olympic Games } \\
\text { in Beijing, 2008 }\end{array}$ \\
\hline Number of people of one-armed take-off & 4 & 9 \\
Number of people of two-armed take-off & 8 & 3 \\
Highest scores & $2.35 \mathrm{~m}$ & $2.36 \mathrm{~m}$ \\
Average scores & $2.268 \mathrm{~m}$ & $2.280 \mathrm{~m}$ \\
\hline
\end{tabular}

It can be seen from table 1 that as for the take-off movement, athletes tends to adopt the one-armed movement. In Sydney Olympic Games in 2000, an athlete of high jump from Russia named Sergey Kliugin won the championship by 2.35 meter, while Andrey Silnov from the same country won the championship by 2.36 meter in Beijing Olympic Games in 2008. These two athletes both adopted the one-armed take-off. By comparing these two terms of Olympic Games, it is obvious that the average scores of high jump in Beijing Olympic Games are higher than that of Sydney Olympic Games. Thus, it is clear that the way of high jump influences the scores (regardless of other factors).

\section{The biomechanical analysis of the one-armed and two- armed take-off in Fosbury Flop}

\section{A. The experimental determination of the relevant data on} one-armed and two-armed take-off

Experimental subject: 15 male students aged 19 to 20 from physical education department that are all professional athletes with good health condition

Experimental apparatus: a domestic three- dimensional measurement, a YA-506B strain-gauge measurement, a tickertimer

Test procedures: The whole experiment was conducted in the laboratory of sports biomechanics. Before the experiment, the subjects were asked to register their information and wear appropriate clothes and practice for several times. Then, the subjects were divided into two groups, of which one group of subjects started their take-off with one arm while the other with both arms. Finally, these two groups were tested respectively.

Data processing: Microsoft EXCEL and the software that can transform the data collected by ergo graph into text file are used. The ticker-timer is used to measure the instantaneous speed in order to find out the magnitude of strength acting on the vertical direction and to record the reverent time when the subjects start their take-off. Then, the strength value, speed and some relevant average values should be counted.

$B$. The analysis on physical strength of one-armed and twoarmed take-off.

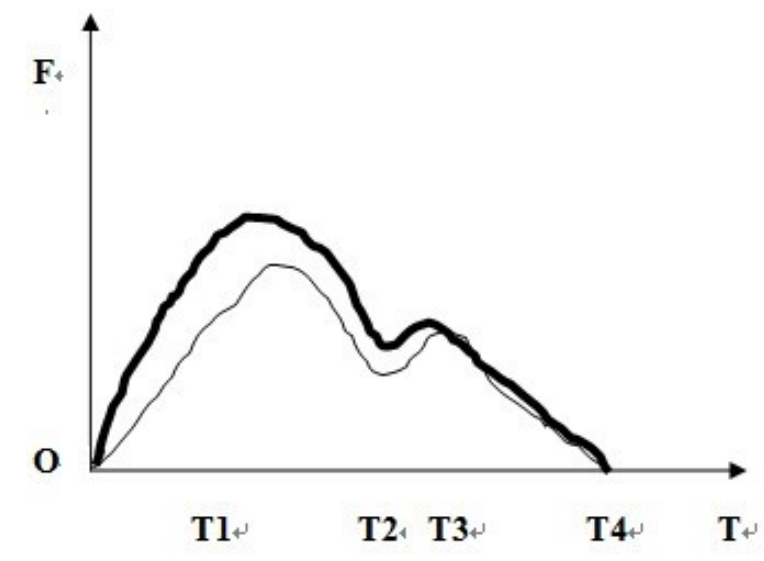

DIAGRAM 1 the analysis on vertical force curve of the take-off

Note: : The vertical force curve of one-armed take-off is shown by the fine line and the vertical force curve of two armed take-off is shown by the thick line. The length of time of these two types of take-off is the same.

The horizontal ordinate indicates time and the ordinate indicates the magnitude of strength, while the letter $\mathrm{O}$ bodyweight. T1 indicates the specific point in time when the maximum force is recorded as the athlete treads on the pedal instrument. T2 shows the instant when the athlete jump to the peak and thus the speed is zero. The period of time from T1 to $\mathrm{T} 3$ is the length of time when the athlete rise high into the air and the vertical force decreases rapidly at this time. T3 shows the instant when the athlete falls down to the pedal instrument and T4 indicates the specific point in time when the force pressed to the instrument equals the body-weight of the athlete. The period of time from T3 to T4 was the buffer time.

TABLE 2 the comparison of the average magnitude of strength between produced respectively by one-arm and two arms on the vertical direction

\begin{tabular}{lc}
\hline & average value of F1 \\
\hline One-armed take-off & $3.157(15)$ \\
Two-armed take-off & $3.685(15)$ \\
\hline
\end{tabular}

From diagram 1 and table 2, it can be concluded that both the maximum value and the average value of the strength 
produced on the vertical direction when the athlete adopts the one-armed take-off are smaller than that produced when twoarmed take-off is adopted. The difference is obvious.

\section{The analysis on the speed of take-off of one-armed and two-armed}

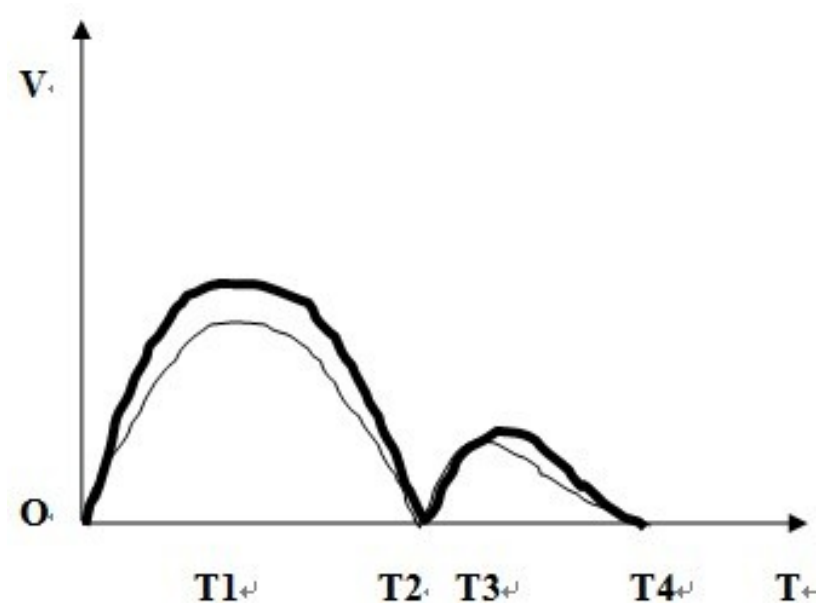

Diagram 2 the curve analysis of the speed on vertical direction when the take- off happens

Note: The velocity curve of one-armed take-off is shown by the fine line and the velocity curve of two armed take-off is shown by the thick line. The length of time of these two types of take-off is the same

The horizontal ordinate indicates time and ordinate indicates the speed of jumping up while Letter $\mathrm{O}$ means the speed of jumping up when the athlete treads the pedal. T1 indicates the time when the speed reaches its maximum as the feet leave the instrument, and T2 indicates the instant when the speed of jumping up gets zero. The period of time from T1 to $\mathrm{T} 2$ indicates the processing time when the athlete jumps in to the air before landing. T3 indicates the instant when the instantaneous speed when the athlete lands on the instrument reaches it's maximum. The period of time from T2 to T3 shows the processing time when the athlete falls down, and T4 means the instant when the body stays still while the period of time from T3 to T4 is the time of buffer stage when the athlete lands.

TABLE 3 the comparison on the average values of the maximum speed produced respectively by one-arm and two arms on the vertical direction

average value of V1

\begin{tabular}{ll}
\hline One-armed take-off & $6.355(15)$ \\
Two-armed take-off & $6.205(15)$ \\
\hline
\end{tabular}

From diagram 2 and table 3, it can be concluded that the average value of the maximum speed produced on the vertical direction when the athlete adopts the one-armed take-off are greater than that produced when two-armed take-off is adopted. The difference is obvious.

\section{The Influence of Strength and Speed on the Performance of Fosbury Flop}

There are two types of take-off of the modern Fosbury Flop, including speed type and strength type. The speed type is characterized by high speed of run-up, short take-off time (the take-off time is from 0.120 s to $0.200 \mathrm{~s}$ ), narrow range and less strength, while the strength type is on the contrary. It can be seen from diagram 1 and table 2 that it consumes more strength to take off by one arm than by both arms on the vertical direction. Thus, the back style high-jump taking off by two arms is called strength type high-jump. From diagram 2 and table 3 , it can be seen that the speed of one-armed take-off is much faster than that of two-armed take-off, and this back style high-jump with one-armed take-off is called speed type high-jump.

Combining table 1, we can see that the athletes tends to adopt the speed type Fosbury Flop. The average scores of high-jump of Beijing Olympic Games in 2008 are $2.280 \mathrm{~m}$ with more athletes adopting one-armed take-off (the speed type Fosbury Flop) while in Sydney Olympic Games in 20002.268m with more athletes adopting two-armed take-off (the strength type Fosbury Flop) The average scores of speed type Fosbury Flop are much better than the strength type Fosbury Flop (without other interference factors). Under some certain conditions, speed is more conducive to improve the scores of high jump.

\section{Conclusion and suggestions}

Speed quality and strength quality are indispensable factors that influence athletes' performance. The coach should attach great importance to not only the technical training, but also the improvement of speed and strength quality.

Speed quality has much more influence on the performance of high jump than strength quality. The athletes should pay more attention to the training of speed quality so as to get ideal performance with the perfect combination of speed and strength.

\section{References}

[1] Chen Xiaozhi. The research reviews of speed strength training. Journal of information science and technology (academic studies), 2008 (15)

[2] Chen Song and Ma Qiwei. The comparative study of the training methods on the quantitative control of speed - its influence on weightspeed curve. Journal of Beijing sport university, 1995 (03)

[3] Zhang Yunai,Ding Guanshan and Yao Gongyan. The key factors that influence the improvement of performance of the running athletes in common colleges and universities - speed strength training. Journal of shandong sports science. 1996 (3)

[4] Wan Deguang and Wan Meng compiled. Modern strength training. People's sport publishing house, 2003

[5] Tian Maijiu. Sports training science. People's sport publishing house, 2000 